\title{
Bioethanol Production from Lignocellulose Waste: A Comparative Study Between First and Second Generation Substrate
}

\author{
Vishal Kumar ${ }^{1 *}$ and Rosy Kataria ${ }^{2}$ \\ ${ }^{1}$ Uttaranchal (Pg) College of Biomedical Sciences and Hospital, Dehradun \\ ${ }^{2}$ Codon Biotech Pvt Ltd, Noida Sector, Noida, UP \\ *Corresponding Author: Vishal Kumar, Uttaranchal (Pg) College of Biomedical Sciences and Hospital, Dehradun.
}

Received: October 17, 2019; Published: November 05, 2019

DOI: 10.31080/ASMI.2019.02.0424

\begin{abstract}
One of the greatest challenges of twenty-first century is to meet the growing demand of energy for transportation, heating and industrial processes, and to provide raw materials for chemical industries in sustainable ways. Biofuels have emerged as an ideal option to meet these requirements in a sustainable manner. Ethanol derived from biomass is the only liquid transportation fuel that does not contribute to the greenhouse gas effect Ethanol production process only uses energy from renewable energy sources; no net carbon dioxide is added to the atmosphere, making ethanol an environmentally beneficial energy source. Most of the first generation biofuels are sourced from crop plants as energy-containing molecules like sugars, oils and cellulose. They provide only limited biofuel yields and have a negative impact on food security. Second generation biofuels are produced from non-food crops including the waste from food crops, agricultural residue, wood chips, and waste cooking oil. Second-generation biofuel feedstock is the nonedible by-product of food crops. There are advantages to using the inevitable by-product of the agricultural industry for biofuel production; no additional fertilizer, water, or land are required to grow this feedstock.

Introduction: One of the greatest challenges of twenty-first century is to meet the growing demand of energy for transportation, heating and industrial processes, and to provide raw materials for chemical industries in sustainable ways. Biofuels have emerged as an ideal option to meet these requirements in a sustainable manner. Bioethanol is presently the most abundant biofuel for automobile transportation. The most appropriate bioethanol technology for the nation would be to produce it from lignocellulose biomass such as rice straw, rice husk, wheat straw, municipal waste and forest waste. Lignocellulose biomass-derived second-generation biofuels are promising alternatives to petroleum-based fossil fuels. The utilization of agricultural residues and wastes for bioethanol production is a cost-effective and environmental-friendly approach for sustainable development. The utilization of agricultural residues and wastes for bioethanol production is a cost-effective and environmental-friendly approach for sustainable development. Considering the recent research progress in the fields of enzyme production, pre-treatment, as well as metabolic engineering of yeasts, and production of bioethanol from lignocellulose agricultural wastes will certainly prove to be a feasible technology to achieve energy security in very near future.

Methods: Yeast isolates were used for the production of ethanol using lignocellulose biomass by using enzyme degradation method. Effective conversion of biomass into ethanol was achieved by the action cellulolytic fungi. After proper isolation, identification and characterization, ethanol production was assessed. Very inexpensive and easily available raw material were used as fermentation media.

Results: After fermentation for four days at $25^{\circ} \mathrm{C}$ and $100 \mathrm{RPM}$, it was observed that ethanol production from bagasse was $4.76 \mathrm{~g} / \mathrm{ml}$, from marigold it was $26.0 \mathrm{gm} / \mathrm{ml}$ and from mango peel it was $26.32 \mathrm{gm} / \mathrm{ml}$.

Conclusions: This study suggested that the agriculture and kitchen waste serve as raw material for ethanol production.

Keywords: Bioethanol; Lignocellulose; Second Generation Biofuels
\end{abstract}

\section{Introduction}

Bioethanol is presently the most abundant biofuel for automobile transportation. It is produced from fermentation of sugars obtained from biomass, either in the form of sucrose, starch or lignocellulose. Today the fuel market dominates the market for ethanol.
In the last quarter century focus has lain on producing fuel ethanol as a substitute or additive to gasoline. Ethanol contains 35\% oxygen that helps complete combustion of fuel and thus reduces particulate emission that poses health hazard to living beings. The toxicity of the exhaust emissions from ethanol is lower than that 
of petroleum sources. Lignocellulose biomass-derived secondgeneration biofuels are promising alternatives to petroleum-based fossil fuels.

First generation bioethanol feedstock come from agricultural cereal and sugar crops that are also sources of human (and animal) food. The bioethanol produced by fermentation of sugars such as sugarcane, sugar beet, sorghum, whey and molasses and starchy feedstock such as grains viz. maize, wheat, root crops such as cassava are commonly known as first generation bioethanol. Sugar crops need only a milling process for the extraction of sugars to fermentation (not requiring any step of hydrolysis), becoming a relatively simple process of sugar transformation into ethanol. In this process, ethanol can be fermented directly from cane juice or beet juice or from molasses generally obtained as a by-product after the extraction of sugar.

Second-generation bioethanol refers to fuel alcohol produced from non-food biomass sources, such as lignocellulose, the most abundant form of carbon on the earth. Agricultural residuals or by-products are annually renewable, abundantly available and account for more than 180 million tons per year. These lignocellulose biomass includes woody material, straws, agricultural waste and crop residues. Second-generation biofuels are expected to reduce net carbon emission, increase energy efficiency and reduce energy dependency, potentially overcoming the limitations of first-generation biofuels. For countries where cultivation of energy crops for bioethanol is difficult, lignocellulose biomass offers an attractive option. The other major benefits of switching to cellulosic ethanol are its renewable nature, long term sustainability, low net carbon emission, high energy efficiency, low energy dependency, increase in national security and diversifying rural economies.

In India, ethanol is mainly produced from sugarcane molasses but the substrate has to compete with the food demand and therefore cannot supply the required amount of ethanol. Therefore, the nation needs to develop bioethanol technologies, which use biomass feedstock that does not have food or feed value. The most appropriate bioethanol technology for the nation would be to produce it from lignocellulose biomass such as rice straw, rice husk, wheat straw, sugarcane tops and bagasse, municipal waste and forest waste.

Ethanol derived from biomass is the only liquid transportation fuel that does not contribute to the greenhouse gas effect.

\section{Material and Methods}

Sample collection and isolation

Different types of yeasts were collected viz. S.cerevisiae, S.boulardii, they were grown on YPD agar (Yeast Peptone Dex- trose) media composing $1 \%$ yeast extract, $2 \%$ peptone, $2 \%$ glucose, and desired volume of distilled water. The cultures were plated on this media and were incubated at $25^{\circ} \mathrm{C}$ for 48 hours. Then the slant was prepared and preserved at $4^{\circ} \mathrm{C}$ for preservation. It was further maintained by sub -culturing.

A slide was prepared from different yeast samples and was observed under compound microscope to observe the cell morphology.
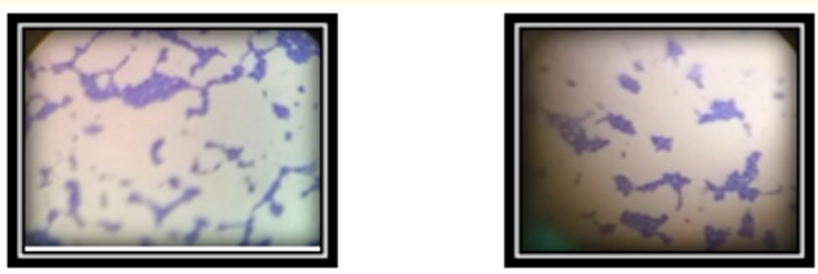

Figure 1 and 2: Yeast culture 1 and 2.

\section{Biochemical characterization of yeast}

Carbohydrate test (sugar fermentation test)

The carbohydrate fermentation test is used to determine whether or not bacteria can ferment a specific carbohydrate. It test for the presence of acid and /or gas produced from carbohydrate fermentation. Basal medium containing a single carbohydrate source such as Glucose, Lactose, Sucrose or any other carbohydrate is used for this purpose.

\section{Acetate utilization test}

Acetate utilization test is used to determine if an organism can use acetate as the sole source of carbon. If so, breakdown of the sodium acetate cause the $\mathrm{pH}$ of the medium to shift toward the alkaline range, turning the colour from green to blue.

\section{Starch hydrolysis test}

Starch molecules are too large to enter into the fungal cells, so some fungi will secrete exoenzyme that will degrade starch into subunits that can be then easily utilized by the organism. Starch agar is a simple nutritive medium with starch added. Since no colour change occurs in the medium when organisms hydrolyse starch, iodine solution is added to the plate after incubation. Iodine turns blue; purple, in the presence of starch. A clearing around the bacterial growth shows that the organism has hydrolysed starch.

\section{Substrate preparation}

Different lignocellulose waste were collected like bagasse, mango peel, and marigold. The above substrate were dried properly so as to remove the water content and then were finely crushed using grinder. After that, the sub stra were e treated with dilute acid 
to hydrolyse the lignin and cellulose present. $\mathrm{pH}$ was set to 5.5-6 then were further given heat treatment at $121^{\circ} \mathrm{C}$ for 15 minutes to ensure complete hydrolysis of cellulose.
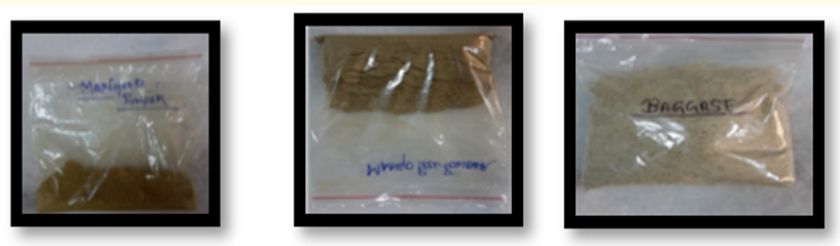

Figure 3

Fermentation media preparation

Yeast subculture derived from 24-48 hours old streak plates were inoculated into $10 \mathrm{~mL}$ YPD media and then were incubated for 24 hours. After incubation, the obtained culture were added to the prepared substrate $(50 \mathrm{ml})$ in a $250 \mathrm{ml}$ conical flask and was kept in incubator shaker at $25^{\circ} \mathrm{C}$ at $100 \mathrm{RPM}$.

Estimation of ethanol produced

After the fermentation of lignocellulose waste the ethanol produced was estimated by two methods.

1. Potassium dichromate method: This method is a preliminary method which gives higher alcohol readings as compared to actual estimation done.

2. Gas chromatography method: The estimation of alcohol can be done by Gas Chromatography (GC) also. Gas chromatography uses a very small amount of sample to detect the actual amount. Thus Gas chromatography estimations are highly accurate. Gas chromatography was used to estimate and detect the amount of bioethanol produced for 3-4 days. The concentration of ethanol produced is shown as peak area and after the total amount of ethanol is calculated in gm/litre. Relevant GC peaks and tables are shown to clarify the actual production as well as related ethanol production utilizing the different lignocellulose wastes.

Formula for calculation of gm/l ethanol produced

$\mathrm{g} / \mathrm{l}$ ethanol $=\underline{\text { Molecular weight of injected sample X Area of sample }}$ Area of standard

Result

\begin{tabular}{|l|c|c|c|}
\hline Sample (1M) & $\begin{array}{c}\text { Amount of } \\
\text { sample( } \boldsymbol{\mu l})\end{array}$ & OD & $\begin{array}{c}\text { Amount of } \\
\text { alcohol (\%) }\end{array}$ \\
\hline Bagasse & 500 & 0.32 & $4.5 \%$ \\
\hline Marigold & 500 & 0.24 & $11.4 \%$ \\
\hline Mango peel & 500 & 0.01 & $14 \%$ \\
\hline
\end{tabular}

Table 1: Showing ethanol estimation by Acid Dichromte Test.

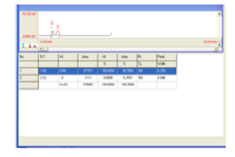

GCRESULT OF BAGASSE

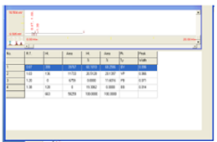

GC RESTLT OF MARIGOLD

Figure 4

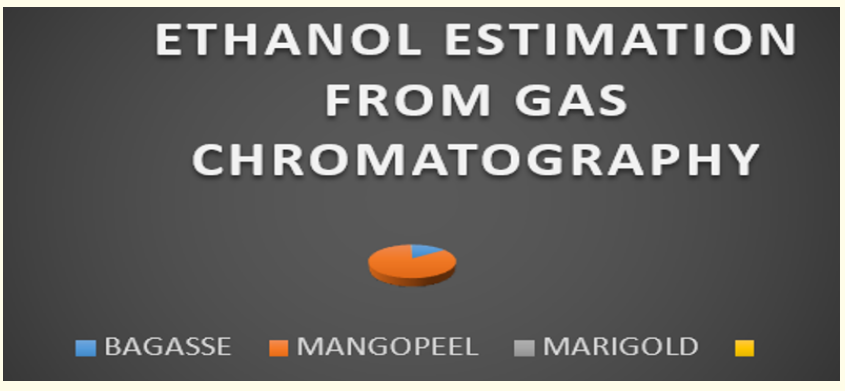

Figure 5
GC Result of bagasse GC result of marigold GC result of marigold

After gas chromatography, it was observed that marigold and mango peel produced comparatively high concentration of bioethanol than that produced by sugarcane bagasse [1-5].

\section{Conclusion}

It can be concluded that second generation lignocellulose waste can yield higher concentration of ethanol than first generation Lignocellulose biomass-derived second-generation biofuels are promising alternatives to petroleum-based fossil fuels. The utilization of agricultural residues and wastes for bioethanol production is a cost-effective and environmental-friendly approach for sustainable development. Considering the recent research progress in the fields of enzyme production, pre-treatment, as well as metabolic engineering of yeasts, production of bioethanol from lignocellulose agricultural wastes will certainly prove to be a feasible technology to achieve energy security in very near future.

\section{Bibliography}

1. Giorgos Markou., et al. "Bioethanol Production by Carbohydrate-Enriched Biomass of Arthrospira (Spirulina) platensis" (2013): 3937-3950.

2. Njoku Hinrich Uellendahl and Birgitte K Ahring. "Biofuels and biomaterials production in a biorefinery - key features for sustainable and economically viable concepts Stephen I.

3. Kang., et al. "Bioethanol from Lignocellulosic Biomass: Current Findings Determine Research Priorities by Qian". The Scientific World Journal (2014): 298153. 
4. O' scar J Sa'nchez and Carlos A Cardona "Trends in biotechnological production of fuel ethanol from different feedstocks". Bioresource Technology 99.13 (2007): 5270-5295.

5. Ahmed Ahmed M., et al. "Bioethanol fuel production from rotten banana as an environmental waste management and sustainable energy Hossain". African Journal of Microbiology Research 5.6 (2011): 586-598.

Volume 2 Issue 12 December 2019 (C) All rights are reserved by Vishal Kumar and Rosy Kataria. 